\title{
A price mechanism for supply demand matching in local grid of households with micro-CHP
}

\author{
G. K. H. Larsen ${ }^{1,1}$, N. D. van Foreest ${ }^{2}$, and J. M. A. Scherpen ${ }^{1}$ \\ ${ }^{1}$ Discrete Technology and Production Automation, Faculty of Mathematics and Natural Sciences, \\ University of Groningen, The Netherlands \\ ${ }^{2}$ Operations, Faculty of Economics and Business, University of Groningen, The Netherlands
}

\begin{abstract}
This paper describes a dynamic price mechanism to coordinate eletric power generation from micro Combined Heat and Power (micro-CHP) systems in a network of households. It is assumed that the households are prosumers, i.e. both producers and consumers of electricity. The control is done on household level in a completely distributed manner. Avoiding a centralized controller both eases computation complexity and preserves communication structure in the network. Local information is used to decide to turn on or off the micro-CHP, but through price signals between the prosumers the network as a whole operates in a cooperative way.
\end{abstract}

\section{Introduction}

The future smart power grid is a complex system with many new challenges. One of the challenges, from a control point of view, is the distributed optimal control with the goal to balance the power supply and demand in the network. The smart grid consists of end-users that are both producers and consumers of electric power (prosumers). Examples of prosumers are a household, a factory or a hospital. The prosumers will have smart meters and home energy management systems installed. In this way, the prosumer can measure his power load at all time. The energy management system is a box where the user can fill in his comfort specification, see [1]. Then based on prices in the network, the box controls the controllable devices for the prosumer. It is clear that the prices affect the decisions for when the prosumers produce and consume electric power. However, the production and consumption of electric power also affects the prices. If it is a network goal to balance supply and demand, the price should rise when the imbalance rise and stay constant when balance is achieved. Therefore, we want a method where both prices and control actions have mutual dependence.

Here we will take the micro-CHP as the example for the device that we are able to control inside each prosumer. This could however also have been a flexible demand side device, such as the washing machine. The micro-CHP systems fueled on gas are of particular interest in countries like the Netherlands where the gas grid is dense. A micro-CHP unit produces heat and electricity at the same time. The market potential is considered to be high, as argued in [2] which considers the potential effects in a grid with a high percentage of domestic distributed generation. The power

1 e-mail : g.k.h.larsen@rug.nl. Special thanks to Ms. D. Alkano and Mr. A.D. Pietersma for their help in finalizing this piece. This work was carried out as part of the Flexines project. 
output of the micro-CHP can be controlled, even though it is subject to several operational constraints.

We design a model that describes the balance of electricity production and consumption in a multi-prosumer Smart Grid. One strategy to achieve balance in the network is to match production and consumption only inside each prosumer. However, it is clear that if neighbors cooperate and share some information with each other, the balancing could be done more efficiently. Imagine prosumer $\mathrm{Y}$ has a high demand, but no possibility to further ramp up his production. Prosumer $\mathrm{Z}$ is using her micro-CHP system to cover heat demand, but has excess power production. Prosumer $\mathrm{Z}$ could earn money by selling electricity to neighbor $\mathrm{Y}$, while the power is still locally produced and consumed in the network.

With this motivation, we propose a communication structure in the network. We aim to achieve cooperation of a large number of spatially connected households. The information to be shared in the final algorithm is price signals and imbalance between prosumers that are connected in a communication graph. The structure of the communication graph is a design choice, and does not need to be the same as the physical power transmission network graph. We will not aim for an auction to achieve balance in the network, but rather let the price signals be driven by the local imbalance. This is because the global information complexity from millions of prosumers is too high, in particular if one also want to take into account transportation cost in the network. The aim in this paper is to provide a fair price for the prosumers in the network.

Using a distributed Model Predictive Control (MPC) approach based on dual decomposition as described in [3] we can achieve balance in the network. Dynamic prices are used to coordinate the prosumers in the network given an information structure.

Preliminary results of our work were presented in [4] and [5]. In [4], dynamic price mechanisms were introduced to coordinate decisions. However, the model still lacked the inclusion of constraints that are inherently present when using micro-CHP's. In [5], the results of [4] were extended to a fully distributed MPC setting via dual decomposition and gradient iterations as presented in [3]. In [6] we presented an extended description of our model and elaborated on the information structure in the network.

In this paper we show a two prosumers network to gain intuition for the variables in the model. And the result for a network of 100 prosumers with realistic demand patterns is shown.

The rest of this paper is organized as follows: Section 2 reviews the dynamic models of the agents in the network that we proposed. Section 3 reviews the distributed MPC technique based on dual-decomposition and sub-gradient iterations. Section 4 focuses on the behavior of the variables in the model with a step-demand. Afterwards, the intuition for the prices in a large network is given. The simulation results for a large network, which verify the proper working of the method, are presented in Section 5. Section 6 concludes the paper with the discussion.

\section{Network model}

In this Section we present our network model. For more details see [5,6]. Each prosumer in the network $i=1, \ldots, n$ has a demand $d_{i}(k)$ that can be measured and a production $p_{i}(k)$ from the micro-CHP, where $k$ is the discrete time. The production is either off

$$
p_{i}(k)=0,
$$

or the production is on, which means that the production can be modulated between a maximum $p_{i, \max }$ and a minimum $p_{i, \min }$ value

$$
-p_{i, \max } \leq p_{i}(k) \leq-p_{i, \min }
$$


Here $p_{i}(k)$ is taken to be opposite in sign to the demand, since we are aiming for network balance. Therefore, $p_{i}(k)$ takes negative values in the model. In order to define our dynamic model for imbalance information in the network, we redefine the variables for demand and production. We introduce the change in demand $w_{i}(k)=d_{i}(k)-d_{i}(k-1)$ and change in production $u_{i}(k)=$ $p_{i}(k)-p_{i}(k-1)$. The imbalance information at prusumer $i$ is then given by

$$
x_{i}(k+1)=A_{i i} x_{i}(k)+\sum_{j \in N_{i}} A_{i j} x_{j}(k)+u_{i}(k)+w_{i}(k)
$$

where $N_{i}$ is the neighbors of prusumer $i$ in the network, $A_{i i}$ weight the prosumers own imbalance and $A_{i j}$ weight neighbor imbalances. Notice that physical changes at the prosumer enters the imbalance information through the terms $u_{i}(k)$ and $w_{i}(k)$. It is the second term on the right hand side that represents influence from neighbors in the network. The important point of this model is that all prosumers couple their notion about imbalance. This is the way that we exchange information in the network, and it will later be the source of dynamic prices in the control algorithm.

We are free to choose information topology in the network independent of the physical power grid. Some restrictions for how to choose the weights $A_{i j}$, we can realize by writing down the model (3) in compact form

$$
\boldsymbol{x}(k+1)=A \boldsymbol{x}(k)+B \boldsymbol{u}(k)+\boldsymbol{w}(k)
$$

where we have defined the vectors $\boldsymbol{x}(k)=\left[x_{1}(k) \ldots x_{n}(k)\right]^{T}, \boldsymbol{u}(k)=\left[u_{1}(k) \ldots u_{n}(k)\right]^{T}$ and $\boldsymbol{w}(k)=\left[w_{1}(k) \ldots w_{n}(k)\right]^{T}$. The $B$ matrix is taken to be the identity matrix because each prosumer only control the micro-CHP that he owns himself. Now the information matrix $A$ defines the information structure in the network. There is a non-zero element in the information matrix only if there is information exchanged between the corresponding nodes. We choose the elements to be nonnegative, since we are dividing notion about the imbalance in the network. In order for the total imbalance in the network to stay constant in time if there are no change in demand or production in the network, it follows that the column sums of the information matrix has to be one. In addition if the information graph is strongly connected it follows that the radial distance of all eigenvectors of the information matrix is less or equal to one. Therefore, we get for free that the uncontrolled system is stable. For more details and proof see [6]. An example of an information structure with a corresponding $A$ matrix is given in Fig. 1.

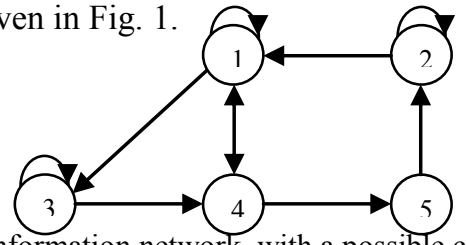

$$
A=\left[\begin{array}{ccccc}
0.3 & 0.5 & 0 & 0.5 & 0 \\
0 & 0.5 & 0 & 0 & 1 \\
0.3 & 0 & 0.5 & 0 & 0 \\
0.3 & 0 & 0.5 & 0 & 0 \\
0 & 0 & 0 & 0.5 & 0
\end{array}\right]
$$

Fig. 1. Here se wee an information network, with a possible corresponding information matrix $A$.

\section{The price mechanism}

In this section we define the optimal control problem and we motivate that we need to solve the problem by the means of distributed Model Predictive Control (MPC). We briefly sketch the approach based on dual decomposition and sub gradient iterations [3]. The dynamic prices follow from the approach.

\subsection{Centralized MPC problem}

The task is to find the optimal input $u_{i}(k)$, i.e. how much to ramp up or down the micro-CHP. First of all, the input is subject to dynamic constraint (3). Secondly, the input is subject to structural 
constraints, which means that the prusumer must make his decision only based on his own information and information from information neighbours. Thirdly, the input is subject to range constraints due to its relation to the production (1) and (2). This is a non-convex constraint because of the gap between zero and $p_{i, \text { min }}$.

Due to the constraints from the micro-CHPs, MPC is a useful technique to solve the optimal control problem, e.g. [7]. This means that the control variables are the solution of an optimization procedure rather than computed by an explicit control law. This is a sub-optimal approach, but we ensure that the range constraints are met for all agents $i$ for all time $k$. In the MPC setting, a modified optimal control problem is solved at each time-step k over a finite horizon $T$. At each time-step $k$ new measurements, predictions of future states and disturbances can be taken into account. The method is widely used in process industry [8].

One of the basic ideas of MPC is the inclusion of models to predict the future dynamics of the system over a finite time horizon. Since the change in production $w_{i}(k)$ is an external signal measured at each time-step $\mathrm{k}$, the formulation needs to provide estimates for $w_{i}(k)$ over the finite time horizon $k, \ldots, k+T$.

We define the cost function that the network as a total try to achieve, subject to the three type of constraints explained in the beginning of this subsection. The goal is to minimize the overall power imbalance in the network as cheaply as possible in $u_{i}(k)$. At each time $k$ there is a quadratic cost associated with the imbalance and control input of each agent $i$. The network cost is the sum of the individual costs, given by

$$
V_{k}=\sum_{i=1}^{n} R_{i i} x_{i}^{2}(k)+Q_{i i} u_{i}^{2}(k),
$$

where the weights $R_{i i}$ and $Q_{i i}$ will reflect the relative importance of each prosumer. By using a higher weight $R_{i i}$ on a hospital for example, it means that it is more important to balance this prosumer to zero. The cost that we want to minimize also includes predictions for the future time horizon $T$, and is given by

$$
\min _{u} V_{T, k}=\min _{u} \sum_{\tau=k}^{T} V_{\tau}
$$

The optimal control inputs are found by minimising this cost over the sequence $\left\{u_{i}(k), \ldots, u_{i}(k+\right.$ $T)\}$. Once the finite optimal control sequence is obtained, we only implement the first input $u_{i}(k)$ to system (3). The horizon is then shifted one sample till $k+1, \ldots, k+1+T$, and the optimization procedure is repeated to obtain $u_{i}(k+1)$.

We now have a solution where the range constraints are satisfied. However, we also have information constraints on $u_{i}(k)$. Solving the above problem requires that one controller knows all information for all prosumers in the network. Therefore, we will present the distributed MPC in the following section.

\subsection{Distributed Model Predictive Control}

Here we briefly explain the result from applying dual-decomposition and sub-gradient iterations. More details can be found in Giselsson and Rantser [3] and [6].

The idea is to replace the neighbour influence in Eq. (3), by a local guess $v_{i}(k)$ of how neighbour will influence the prosumer. Doing so, we need to add the constraints that what we guess about the neighbours has to be the same as the actual neighbour influence. By relaxing these extra constraints using standard Lagrangian method, we introduce a new set of optimization variables $\lambda_{i}(k)$. These are the Lagrange multipliers that are interpreted as prices [9].

Problem (6) is reformulated from a minimization problem to a max-min problem. We achieve a fully distributed method by isolating the terms from each prosumer in the max-min problem and including sub-gradient iterations to obtain the optimal price sequence. 
Each prosumer need to solve the local problem to obtain $u_{i}(k), x_{i}(k)$ and $v_{i}(k)$ over time horizon $T$

$$
\min _{u_{i}} \sum_{\tau=k}^{k+T}\left[V_{i, k}+\lambda_{i}(\tau) v_{i}(\tau)-\sum_{j \in N_{i}} \lambda_{j}(\tau) A_{j i} x_{i}(\tau)\right]
$$

where prices from information neighbours are given, and $V_{i, k}=R_{i i} x_{i}{ }^{2}(k)+Q_{i i} u_{i}{ }^{2}(k)$. Notice that the summation over neighbours in the last term assumes a symmetric $A$, but a symmetric $A$ is no requirement for the method. The prices are updated according to sub-gradient steps

$$
\lambda_{i, r+1}(k+1)=\lambda_{i, r}(k)+\gamma_{i, r}\left[v_{i, r}(k)-\sum_{j \in N_{i}} A_{i j} x_{j, r}(k)\right]
$$

where $r$ labels the sub-gradient iteration, and $\gamma_{i, r}$ is the step size chosen so that the algorithm converge. Notice that the local rules (7) and (8) result from the global optimal control problem.

\subsection{The algorithm}

The algorithm that is implemented to solve the problem is as follows

1. At time-step $k$ : while prices has not converged do:

a. Solve the local optimal control problems (7) at each prosumer, given prices from the neighbors, to obtain the control inputs $u_{i}(k)$.

b. Update the prices according dynamic equation (8)

2. When price has converged, implement the control to the system and move to $k+1$ to repeat the procedure.

The procedure of iteratively solving point $\mathrm{a}$. and $\mathrm{b}$. we call the price bargaining process.

Because of the non-convex range constraints connected to the production $p_{i}(k)$, the algorithm will in some cases not converge. In this case the algorithm oscillates between turning a micro-CHP off or turning it on. When this happens, we terminate the algorithm and implement the last decision before the oscillation.

\section{A two prosumers example}

In this section we look at a network of two prosumers to gain intuition for the variables in the model, and to verify that the price signals work as we expect.

Suppose that two neighbors $\mathrm{Y}$ and $\mathrm{Z}$ are exchanging information about their imbalance. Each of them weights themselves by a factor 0.8 and their neighbor by a factor 0.2 . $Z$ has a micro-CHP present in her house with unused capacity, while $\mathrm{Y}$ has no opportunity for further ramping up his power production.

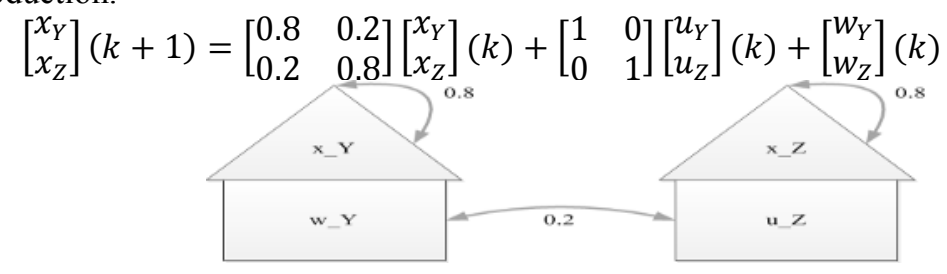

Fig. 2 Two households in a information network.

Fig. 2 shows the corresponding network of Eq. (9). Suppose that the network is in equilibrium, then at time $\mathrm{k}=0 \mathrm{Y}$ turns on his $\mathrm{TV}$ and consumes one unit of power. $\mathrm{Y}$ can himself do nothing to regain 
balance. However, since he shares his imbalance information with $\mathrm{Z}, \mathrm{Z}$ can react and turn on her production. We see in Fig. 3 to the left that if $Z$ does nothing the imbalance of both households shown in stippled lines converge to half a power unit, even if only $\mathrm{Y}$ has a constant demand shown in solid blue line. Fig. 3 to the left shows the situation when $\mathrm{Z}$ ramp up her production according to solution of Eq. (7). Both imbalances in stippled lines converge to zero. Y has a constant demand, shown in solid blue line, and Z's production, shown in dotted red line, converges to a constant production equal to Y's demand. The plots in this section do not include the on-off constraints on the micro-CHP.
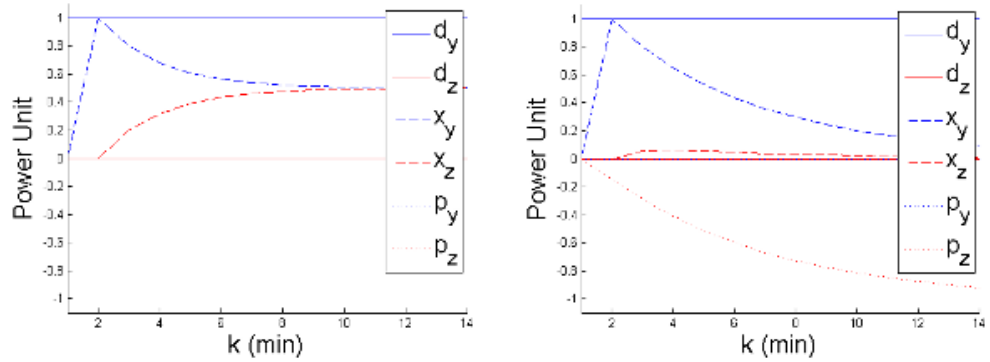

Fig. 3. The left figure shows the imbalance in the network when $\mathrm{Z}$ does not ramp up her production. The right figure shows the result when both prosumers solve the combined problem (7) and (8).
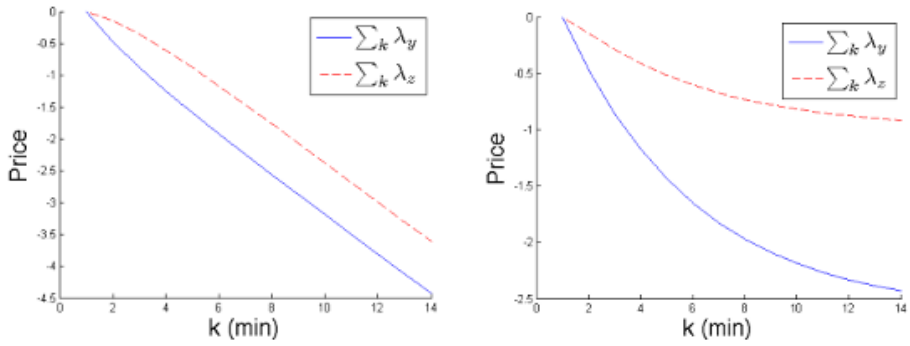

Fig. 4. The left figure shows the prices when $Z$ does not ramp up her production, while the right figure shows the prices when $\mathrm{Z}$ does ramp up her production.

Fig. 4 shows the price signals in the network corresponding to the scenarios shown in Fig. 3. The right figure shows that the prices in the network keep rising when there is an imbalance. The left figure shows that the prices converge to a constant value for both prosumers when the network is back in balance. Prosumer $\mathrm{Y}$ has to pay more than prosumer $\mathrm{Z}$ here, solid line and stippled line respectively.

\subsection{Price behavior in a large network}

In the previous subsection we saw how the prices in the network behave for a step demand of one user. Each prusumer has a unique price depending on the local imbalance, and the dynamic prices follows from the distributed MPC approach. Here we will discuss how the price signal behaves in a large network.

If the imbalance high is high then the price rise quicker than if the imbalance is low. When there is balance between production and demand in the network, the prices do not change. The prices will be highest at the prosumer where the imbalance occurred, and at his close information neighbors. This means that close by information neighbors will have the opportunity to react fastest to an imbalance. By choosing physical close neighbors as information neighbors local production for local demand is therefore stimulated. That the close by information neighbor has the highest price also means that he has the most to earn by producing.

To summarize; price signals spread as a ripple on the water through the connections in the information network. 


\section{Simulation results}

In this section we will test the model presented in Section 2 with the algorithm presented in Section 3 in a network where $n=100$. The local optimization problem is solved by GuRobi 4.6 Mixed Integer Quadratic Program (MIQP). The MIQP is used because of the non-convex on-off constraints of the micro-CHP. We report the scalability of the problem for a centralized solution versus the distributed solution.

\subsection{A neighbourhood with 100 prosumers}

We let the information network have a circular topology, where each prusumer $i=1, \ldots, n$ weight himself with 0.6 and two neighbours with 0.2 . The network and $A$ matrix are shown in Fig. 5.

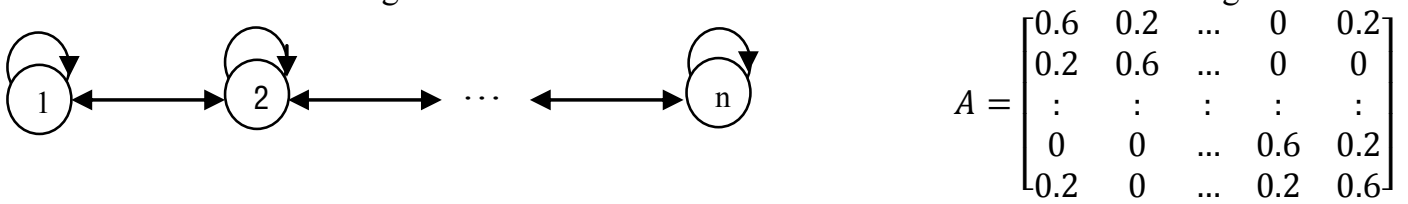

Fig.5 Circular network. Each prosumer has two neighbours, i.e. prosumer 1 and $\mathrm{n}$ are connected.

The production will be allowed to modulate between $0.3 \mathrm{~kW}$ and $1 \mathrm{~kW}$ when it is on, or the output is zero when the micro-CHP is off. This is typical values for the electric output of a microCHP. In addition we require that the micro-CHP is off for at least 15 minutes after it has been turned off, and that it is on for at least 15 minutes after it has been turned on. This represents ramp up and ramp down behaviour, and can easily be exchanged with a more realistic pattern. The important point is that the optimisation procedure anticipates on this behaviour into account while scheduling on and off decisions.

The individual demand patterns are realistic patterns, generated according to [10]. The resolution of the pattern is one minute. In the MPC setting we include predictions for the change in demand. The more accurate notion we have about the future, the better the algorithm will perform. Here we use the measurement of the change in demand $w_{i}(k)$ at time $k$ as prediction for the rest of the prediction horizon $k, \ldots, k+T$. We use a prediction horizon $T=8$ in the simulation.

Fig. 6 to the left shows the result for $n=100$. The stippled line shows the total demand in the network. The dotted line shows the total production resulting from the algorithm presented in Section 3.3. We see that the production-curve mirrors the demand-curve well. As a consequence, the imbalance plotted in solid line, oscillates around zero. The spices can be avoided by a better prediction of the change in demand. However, because of the ramp up constraints and ramp down constraints of the micro-CHP there will always be the case that the network is sometimes a net producer and other times a net consumer.

Fig. 6 to the left shows the situation at one prosumer. We see that the constraints from the microCHP are satisfied. More details about the implementation can also be found in [11].
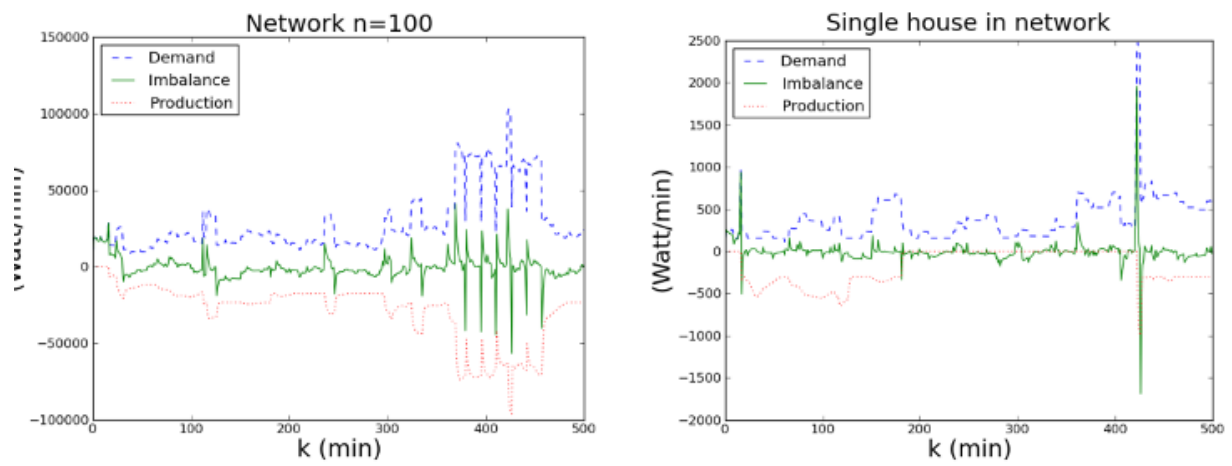

Fig. 6 The result from the network is shown to the left, and the result from one prosumer is shown to the right. 


\subsection{Scalability}

In the distributed implementation each prosumer makes his decision based on local variables. The distributed implementation also scales better than the centralized implementation from a computation point of view. We compare the computation time for $n=5$ and $n=250$ for the distributed and the centralized implementation.

In the distributed case solving the network of 250 prosumers takes about six times as long as solving for the network of 5 prosumers. In the centralized case solving the network of 250 prosumers takes about 30 times as long as solving for the network of 5 prosumers.

\section{Discussion}

We have seen that by embedding the electrical power grid in the framework described in [3], distributed Model predictive Control of decentralized power generation can be achieved. Local actions are coordinated by exchanging prices and imbalance information between prosumers. Following from the method, each prosumer has a unique dynamic price pattern. By choosing physical close neighbors as communication neighbors, local production for local demand is stimulated. If $A$ represents households connected to one low voltage transformer station, the transfer over the transformer-link is minimized. The distributed approach scales better than centralized approach from a computational point of view.

The method leaves design freedom in information topology, production capacity constraints, and relative importance of each prosumer. In this paper the production from a micro-CHP was taken to be the device to be controlled. However, we could equally well aim at coordinating storage devices or demand side devices such as washing machines. Using MPC we can include knowledge about the future situation, and anticipate on this when obtaining prices and on-off actions in the network.

The coupling to a heat-buffer for the heat-output of the micro-CHP was not included. This would serve as additional constraints. We could also use other type of cost functions, taking into account aspects such as economic benefit or climate considerations.

\section{References}

1. http://www.flexines.org/

2. R. Veen, "Balancing market design for a decentralized electricity system: Case of The Netherlands, " in Infrastructure Systems and Services: Building Networks for a Brighter Future, (2008)

3. P. Giselsson, A. Rantzer, "Distributed model predictive control with suboptimality and stability guarantees," in Proc. of the 49:th IEEE Conference on Decision and Control 2010, pp. 7272 - 7277 (2010)

4. G. K. H. Larsen, J. M. A. Scherpen, N. D. van Foreest, E. Doornbos, "Distributed control in a network of households with micro-chp." in Proc. 18th IFAC World Congress, pp. 5320 - 5325 (2011)

5. G. K. H. Larsen, S. Trip, N. D. van Foreest, J. M. A. Scherpen, "Distributed mpc for controlling micro-chps in a network.", in Proc The 2012 American Control Conference (2012)

6. G. K. H. Larsen, N. D. van Foreest, J. M. A. Scherpen "Distributed control of the power supply-demand balance." Submitted (2012)

7. M. Morari, J. Lee, "Model predictive control: Past, present and future," Computers \& Chemical Engineering, 23, 4, pp. 667-682 (1999)

8. E. F. Camacho, C. A. Bordons, Mode/ Predictive Control in the Process Industry. Springer (1997)

9. A. Rantzer, "On prize mechanisms in linear quadratic team theory," in Proc. 46th IEEE Conference on Decision and Control, pp. $1112-1116$ (2007)

10. J. Paauw, B. Roossien, M. Aries, O. Guerra Santin, "Energy pattern generator; understanding the effect of user behaviour on energy systems." First European Conference Energy Efficiency and Behaviour, (2009)

11. S. Trip, "Distributed model predictive control with applications to a network of micro-chps," Master's thesis, University of Groningen, (2011) 